\title{
Propagating phase interface with intermediate interfacial phase: phase field approach
}

Momeni, Kasra, kmomeni@iastate.edu; Levitas, Valery, lowa state university, United States

\begin{abstract}
An advanced three-phase phase field approach (PFA) is suggested for a nonequilibrium phase interface that contains an intermediate phase, in particular, a solid-solid interface with a nanometer-sized intermediate melt (IM). A thermodynamic potential in the polar order parameters is developed that satisfies all thermodynamic equilibrium and stability conditions. The special form of the gradient energy allowed us to include the interaction of two solidmelt interfaces via an IM and obtain a well-posed problem and mesh-independent solutions. It is proved that for stationary 1D solutions to two Ginzburg-Landau equations for three phases, the local energy at each point is equal to the gradient energy. Simulations are performed for $\beta$ ?d phase transformations (PTs) via IM in an HMX energetic material. The obtained energy IM width dependence is described by generalized force-balance models for shortand long-range interaction forces between interfaces but not far from the melting temperature. A force-balance model is developed that describes phase field results even $100 \mathrm{~K}$ below the melting temperature. The effects of the ratios of width and energies of solid-solid and solid-melt interfaces, temperature, and the parameter characterizing interaction of two solid-melt interfaces, on the structure, width, energy of the IM and interface velocity are determined by finite element method. Depending on parameters, the IM may appear by continuous or discontinuous barrierless disordering or via critical nucleus because of thermal fluctuations. The IM may appear during heating and persist during cooling at temperatures well below than it follows from sharp-interface approach. On the other hand, for some parameters when IM is expected, it does not form, producing an IM-free gap. The developed PFA represents a quite general three-phase model and can be extended to other physical phenomena, such as martensitic PTs, surface-induced premelting and PTs, premelting/disordering at grain boundaries, and developing corresponding interfacial phase diagrams.
\end{abstract}

